\title{
Heat of Gasification for Pyrolysis of Charring Materials
}

\author{
MERWIN SIBULKIN \\ Division of Engineering \\ Brown University \\ Providence, Rhode island 02912, USA
}

\begin{abstract}
The relationships between the heats of reaction, pyrolysis and gasification are examined for vaporizing and charring materials.During. burning of thermally thick, vaporizing materials the heat of gasification $\mathrm{h}_{\mathrm{g}} \equiv \dot{\mathrm{q}}_{\mathrm{net}}^{*} / \mathrm{m} \mathrm{G}_{\mathrm{w}}$ is equal to the heat of pyrolysis $h_{p}$. However, no simple relationship exists between $h_{g}$ and $h_{p}$ during the burning of charring materials. Solutions for pyrolysis of cellulose are obtained using a onedimensional, numerical model with material properties estimated from the literature. For this material it is found that after an initial transient $h_{g}$ is about twice $h_{p}$. For a semi-infinite slab the value of $\mathrm{h}_{\mathrm{g}}$ then remains nearly constant; for a finite slab the value of $h_{g}$ may decrease with time to values less than $h_{p}$. Changes in the assumed chemical reaction rate are found to have a minor effect on $h_{\mathrm{g}}$. The heat of gasification is also relatively insensitive to changes in the char density and thermal conductivity. The variation of $h_{g}$ with time is nearly independent of the assumed heat of reaction, but the magnitude of $h$ varies almost directly with the value of the heat of reaction. Changes in the incident heat flux give corresponding changes in the gasification rate, but much smaller changes in the heat of gasification.
\end{abstract}

\section{INTRODUCTION}

One of the modern developments in fire science has been the use of mathematical models. Such models are used to gain a more fundamental knowledge of fire phenomena, to interpret standard fire tests, and (in the future) to design buildings with a prescribed degree of fire safety. Diffusion flame models, e.g. Pagni (1980), Sibulkin et al. (1982), have been developed to predict burning rates and extinction limits. These models have also identified the material properties which most affect burning. Two of these parameters are the heat of combustion and the heat of gasification, and thcir ratio (as used in the B-number for example) is a key factor in burning rate calculations.

Most diffusion flame analyses to date have modeled simple fuels, such as polymethyl methacrylate (PMMA). For these fuels there is no accumulation of char at the fuel surface. Consequently, it can be shown that after an initial transient the surface temperature, heat of gasification and burning rate are independent of time (as long as the fuel remains thermally thick). On the other hand, materials which char (such as wood) show a dependence on time related to the growth of the char layer. We have begun to study the burning of a particularly simple charring material, i.e., cellulose (Sibulkin and Tewari, 1985) and are currently measuring its heat of gasification. To guide us in this study, we have evaluated a onedimensional model for pyrolysis of cellulose, and the results 
are presented in this paper. We first examine the relationships between the heats of reaction, pyrolysis and gasification. Numerical results are then presented for these parameters for cases of constant incident heat flux. The heat flux into the sample is not constant, however, because of radiative heat loss from the surface. ${ }^{1}$

The considerable effort which has been devoted to the kinetics of cellulose pyrolysis (c.g., Lewellen et al., 1977) is not discussed in this paper. A nominal reaction rate is taken from the literature, and the effect of variations in the assumed reaction rate are presented. A similar procedure is used for the still controversial heat of reaction for cellulose pyrolysis. The analysis assumes that the material has been dried (as was done in the burning experiments referenced above). It is also implicitly assumed that no oxygen is present at the surface to react with the char. At present this appears to be the best assumption for surfaces "shielded" by a diffusion flame. Possible surface reactions with carbon dioxide and water vapor are also excluded from consideration; these may become significant at higher temperatures.

\section{HEAT OF GASIFICATION ANALYSIS}

The standard definition for the heat of gasification $h_{g}$ as used in diffusion flame theory is

$\mathrm{h}_{\mathrm{g}} \equiv \dot{\mathrm{q}}_{\mathrm{net}}^{\prime \prime} / \dot{\mathrm{m}}_{\mathrm{G}, \mathrm{w}}^{\prime \prime}$

Its relationship to the heat of pyrolysis is discussed below.

\section{Vaporizing Materials}

We first consider the case of a thermally thick vaporizing fuel as shown in Fig. 1(a). For this case steady burning can occur at a mass loss rate $\dot{m}_{G, w}$ and a surface regression rate $V_{p}=m_{G, W}^{\prime \prime} /{ }_{S}$. By fixing the origin of the $x$ coordinate in the moving surface and using a control volume analysis between $x=0^{-}$and $x=\infty$ (where $x=0^{-}$is in the gas phase just of $f$ the surface), one obtains the energy balance

$\dot{\mathrm{q}}_{\mathrm{net}}^{\prime \prime}=\dot{\mathrm{m}} \dot{\mathrm{G}, \mathrm{w}}^{\prime \prime}\left(\mathrm{h}, \mathrm{w}-\mathrm{h}_{\mathrm{S}, \infty}^{*}\right)$

If one now defines the heat of pyrolysis $h_{p}$ as the difference in total enthalpy between the products of pyrolysis at the surface temperature $T_{W}$ and the virgin material at $T_{\infty}$ (per unit mass of volatile products), one has for a non-charring material

$h_{p} \equiv h_{G, w}^{*}-h_{S, \infty}^{*}$.

Combining Eqs. (1) and (2) shows that for the thermally thick case

$h_{g}=h_{p}$

\footnotetext{
${ }^{1}$ Because of their low boiling points, surface radiation is normally unimportant for liquid fuels. It has been shown to be significant for fuels such as PMMA ( $\mathrm{T}_{\mathrm{w}} \approx 650 \mathrm{~K}$ ), and is even more important for charring materials where $T_{W}=900 \mathrm{~K}$.
} 
For this reason the quanity defined by equation (1) has also been called "heat of pyrolysis" in the literature (which is a source of confusion).

An alternate definition of the heat of pyrolysis for vaporizing materials is obtained as follows. The relationship between the total and sensible enthalpies of species $i$ at temperature $T$ is given by

$h_{1, T}^{*} \equiv h_{1, \infty}^{*}+h_{i, T} \equiv h_{1, \infty}^{*}+\int_{T_{\infty}}^{T} c_{p, i} d t$

and the heat of reaction is $-h_{r, \infty}=h_{G, \infty}^{*} h_{S}{ }^{*}$. Using these definitions, Eq. (3) becomes

$h_{p}=\left(-h_{r, \infty}\right)+h_{G, w}$

(Here the convention has been used that $h_{r, \infty}$ is positive when exothermic while $h_{p}$ is positive when endothermic.) For a material such as PMMA, $h_{p}$ is slightly dependent on the burning rate through the dependence of $T_{w}$ on burning rate.

\section{Charring Materials}

The comparable analysis for a thermally thick, charring material as shown in Fig. (b) is more complicated. The material is assumed to be dry and to maintain its orginal geometry. We begin by assuming a pyrolysis process of the form

Active Solid $(\sigma) \rightarrow$ Gaseous Volatiles $(G)+$ Char $(k)$.

During pyrolysis the bulk density $\rho_{\sigma}$ of the active solid decreases from a value $\rho_{S}$ for the virgin solid to zero, while the char bulk density $\rho_{k}$ increases from zero to its final value $\rho_{\mathrm{C}}$. The density of gaseous products is neglected so that during pyrolysis

$\rho=\rho_{\sigma}+\rho_{k}$

and it is hypothesized that

$\frac{d \rho_{k}}{d t}=-\frac{{ }^{\rho} C}{\rho_{S}} \frac{d \rho_{\sigma}}{d t}$

Manipulation of Eqs. (7) and (8) gives the relations

$\frac{d \rho_{\sigma}}{d \rho}=\frac{\rho_{S}}{\rho_{S}-\rho_{C}}$ and $\frac{d \rho_{K}}{d \rho}=-\frac{{ }^{\rho} C}{\rho_{S}{ }^{-} \rho_{C}}$

which are used subsequently.

Applying conservation of mass to a differential element of length $\mathrm{dx}$ and unit cross-sectional area gives

$\frac{\mathrm{dm}}{\mathrm{dx}}{ }_{\mathrm{G}}^{\prime}=+\frac{\partial p}{\partial \mathrm{t}}$ 
(where $\dot{\mathrm{m}}_{\mathrm{G}}^{\prime}$ is taken as positive in the negative $\mathrm{x}$ direction).

Applying conservation of energy to the same element gives

$\frac{\partial}{\partial t}\left(\rho_{\sigma} h_{\sigma}+\rho_{k} h_{k}\right)=\left[-\frac{\partial \rho}{\partial t}\right] h_{r, \infty}+\frac{\partial}{\partial x}\left[k \frac{\partial T}{\partial x}\right]+\frac{\partial}{\partial x}\left(\dot{m}^{\prime \prime} h_{G}\right)$.

Intergration of Eq. (11) from $x=0$ to $\infty$ gives

$\int_{0}^{\infty} \frac{\partial}{\partial t}\left(\rho_{\sigma} h_{\sigma}+\rho_{k} h_{k}\right) d x=\dot{m}_{G, w}^{\prime \prime} h_{r, \infty}+\dot{q}_{w}^{\prime \prime}-\dot{m}_{G, w}{ }_{G, w}$

where $\dot{\mathrm{q}}_{\mathrm{W}}^{\prime \prime} \equiv \dot{\mathrm{q}}_{\mathrm{n}}^{\prime \prime}$. Expanding the integrand on the LHS of Eq. (12) and using Eq. (9) gives

$\frac{\partial}{\partial t}\left(\rho_{\sigma} h_{\sigma}+\rho_{k} h_{k}\right)=\left(\rho_{\sigma} c_{\sigma}+\rho_{k} \mathcal{c}_{k}\right) \frac{\partial T}{\partial t}+\left[\left(\frac{\rho_{S}}{\rho_{S}-\rho_{C}}\right] h_{\sigma}-\left[\frac{\rho_{C}}{\rho_{S}^{-\rho} C}\right] h_{k}\right] \frac{\partial \rho}{\partial t}$.

Substitution of Eq. (13) into Eq. (12) and application of Eq. (1) gives as the general result for the heat of gasification

$$
\begin{aligned}
& h_{g}=\left(-h_{r, \infty}\right)+h_{G, w}+\frac{1}{\dot{m}_{G, w}^{\prime \prime}} \int_{0}^{\infty}\left[-\frac{\partial \rho}{\partial t}\right]\left[\left[\frac{{ }^{\rho} C}{\rho_{S^{-}{ }_{C}}}\right] h_{k}-\left[\frac{{ }^{\rho} S}{\rho_{S}{ }^{-}{ }_{C}}\right] h_{\sigma}\right] d x \\
& +\frac{1}{\dot{\mathrm{m}}_{\mathrm{G}, \mathrm{w}}^{\prime \prime}} \int_{0}^{\infty}\left(\rho_{\sigma} c_{\sigma}+\rho_{k} c_{k}\right) \frac{\partial T}{\partial t} d x .
\end{aligned}
$$

In Eq. (14) the first integral gives the change in sensible enthalpy between active solid and char while the second integral is the unsteady, energy storage term.

Again using the definition that $h_{p}$ is the difference in total enthalpy between the products of pyrolysis at $T_{w}$ and the virgin material at $T_{\infty}$ (per unit mass of volatiles), one obtains for a charring material

$h_{p}=\left(-h_{r, \infty}\right)+h_{G, w}+\left[\frac{{ }^{\rho} C}{\rho_{S}{ }^{-\rho} C}\right] h_{C, w}$

(which reduces to Eq. (5) for ${ }^{\rho} \mathrm{C}=0$ ).

A comparison of Eqs. (14) and (15) shows that there is no simple relationship between the heat of gasification and the heat of pyrolysis for charring materials. Calculated values for $h_{g}$ and $h_{p}$ are presented in the next section. 


\section{NUMERICAL RESULTS FOR CELLULOSE}

Numerical solutions have been obtained for the propagation of a onedimensional pyrolysis wave into a charring solid. The method was developed by Kung (1972), and a modification of his computer program (Tamanini, 1976) was used. The finite-difference program solves Eqs. (10) and (11) for a pyrolysis reaction given by

$-\frac{\partial \rho}{\partial t}=\rho_{\sigma} A \exp (-E / R T)$.

The material is assumed to be dry and to maintain its original geometry. Results are presented for a symmetrically heated slab having a half-width $L$ which is subjected to a constant, external incident flux $\dot{q}_{\mathrm{ex}}^{\prime \prime}$. Values of $\mathbf{L}=1 \mathrm{~cm}$ and $\dot{\mathrm{q}}_{\mathrm{ex}}^{\prime \prime}=50 \mathrm{~kW} / \mathrm{m}^{2}$, chosen to simulate our previous burning measurements (Sibulkin and Tewari, 1985), are used except as noted. The net heat flux is determined as part of the calculation from

$\dot{\mathrm{q}}_{\text {net }}^{\prime \prime}=\dot{\mathrm{q}}_{\mathrm{ex}}^{\prime \prime}-\epsilon \sigma\left(\mathrm{T}_{\mathrm{w}}^{4}-\mathrm{T}_{\infty}^{4}\right)$.

The set of base values chosen for the properties of cellulose used in the calculations are given in Table 1; they are taken as best estimates from the literature. In particular, the kinetic parameters for cellulose are average values from Lewellen et al. (1977). The properties of cellulose char and both the rate and heat of reaction of cellulose pyrolysis are not well known; they are inter-related and depend on the external heating rate and the scale of the sample. Results are presented for the sensitivity of the heat of gasification to changes in the values of these properties.

The calculated values of surface temperature and net heat flux are shown in Fig. 2. The calculated values for $T_{W}$ in the vicinity of $900 \mathrm{~K}$ agree with measured surface temperatures made during flaming combustion. Of particular significance is the drop in $q_{\text {net }}^{\prime \prime}$ to about 20 percent of the incident heat flux. The calculations were terminated when 1 percent of the material at the centerplane was pyrolyzed.

Profiles of density and temperature within the material are shown in Fig. 3. Pyrolysis occurs in a fairly narrow zone which increases from $1 \mathrm{~mm}$ to $2 \mathrm{~mm}$ as the wave moves inward. The temperature range for which 90 percent of pyrolysis takes place is shown by the solid segments of the temperature curves; the range of values is about $600-750 \mathrm{~K}$. Note that the temperature at the centerplane has already begun to rise at $t=40 \mathrm{~s}$, so that after this time the slab is not thermally thick.

Typical curves showing the time variation of pyrolysis rate $\mathrm{V}_{p} \equiv \dot{m}_{\mathrm{G}_{4} w} /\left(\rho_{\mathrm{S}^{-\rho}} \rho_{C}\right)$ and heat of gasification $h_{g}$ given by the calculations are presented in Fig. 4 . We have chosen to plot the pyrolysis rate in terms of $V_{p}$ since one can interpret the numerical values more easily, e.g., $V_{p} \times 10^{5}(\mathrm{~m} / \mathrm{s})=1$ is a velocity of $1 \mathrm{~mm}$ in $100 \mathrm{~s}$. Gasification begins after about $5 \mathrm{~s}$, reaches a maximum at about $20 \mathrm{~s}$ and then begins to decrease. During this period the variation of $h_{g}$ is approximately the inverse of $V_{p}$ (see Eq. 1). Using a simplified, analytical model for pyrolysis, Delichatsios and deRis (1984) find that in the "final" period $V_{p} \propto t^{-1 / 2}$, and these numerical calculations give a similar result when the centerplane temperature remains at $T_{\infty}$ i.e. for $L=\infty$. For the case of $L=1$ $\mathrm{cm}$, the pyrolysis rate departs from the $L=\infty$ curve at about $70 \mathrm{~s}$ and then begins a rise which is a consequence of the increasing centerplane temperature. After the initial transient, $h_{g}$ is nearly constant for the $L=\infty$ case which would simplify the problem of modeling the complete (gas plus solid phase) diffusion flame. However, once the thermal wave reaches the centerplane of the material, the value of $h_{g}$ decreases as shown by the $\mathrm{L}=1 \mathrm{~cm}$ case. 
A comparison of the heats of reaction, pyrolysis and gasification is shown in Fig. 5 for the base case. The value of $\left.\left(-h_{r},\right)^{2}\right)$ is constant at an assumed value of $0.5 \times 10^{+6} \mathrm{~J} / \mathrm{kg}$. The value of $\mathrm{h}_{\mathrm{p}}$ calculated using Eq. (15) is 3 to 4 times greater than $h_{r \infty}$; it increases with time as $T_{w}$ increases. At $t=50 \mathrm{~s}$, the value of $h_{g}$ is about twice that for $h_{p}$ but becomes less than $h_{p}$ after about $150 \mathrm{~s}$. This difference in behavior of $h_{p}$ and $h_{g}$ emphasizes the importance of the unsteady aspects of pyrolysis of charring materials.

Considerable effort has gone into measuring the overall reaction rate for cellulose pyrolysis. A comparison of the kinetic parameters obtained by several investigators is given in Lewellen et al. (1977); over the temperature range $600-750 \mathrm{~K}$ where most of our pyrolysis occurs the variation in the reported reaction rates is less than a factor of 10. The sensitivity of the calculated values of $h_{g}$ to such a change in the pre-exponential factor $A$ is shown in Fig. 6. It is found that factor of 10 changes in reaction rate have only a modest effect on $h_{g}$. Thus use of the current literature values should be satisfactory.

The density of cellulose char is known to depend on the rate of heating and on impurities in the material, which can be either natural minerals or artificial fire retardants. Values of the thermal conductivity of char are also uncertain but should tend to decrease as the char density decreases. The sensitivity of $h_{g}$ to a decrease in the product ${ }^{\rho} \mathrm{C}^{k} \mathrm{C}$ by a factor of 4 is shown in Fig. 7 . Fortunately, $\mathrm{h}_{\mathrm{g}}$ is relatively insensitive to changes in this parameter.

Widely varying values for the magnitude, and even the sign, of the heat of reaction for cellulose pyrolysis have been reported (Kanury and Blackshear, 1970). Some of these variations depend on whether the results are for an inert or oxidizing atmosphere, whether the samples were dried or at a specified relative humidity, and whether or not the results have been corrected to a standard temperature (Kung and Kalelkar, 1973). On Fig. 8 the effect on $h_{g}$ of varying $h_{r, \infty}$ from 0 to 2 times the base value is presented. The variation of $h_{g}$ with time is nearly independent of $h_{r}$. but the magnitudes of $h_{g}$ differ by about $3 / 4$ of the change in $h_{r, \infty}$ Further experimental results are needed to resolve this uncertainty. (Direct measurements of $\mathrm{h}_{\mathrm{g}}$ for cellulose are in progress at our laboratory.)

The effects of changing the incident heat flux by up to a factor of 2 are examined in Figs. 9 and 10 . Both the initial pryolysis delay and its peak value are seen in Fig. 9 to be strongly dependent on $\mathrm{q}_{\mathrm{ex}}^{\prime \prime}$. This is followed by a decreased sensitivity to $q_{e x}^{\prime \prime}$ after $t=100 \mathrm{~s}$. The results for $h_{g}$ given in Fig. 10 show a similar dependence of the duration of the initial transient on $\dot{q}_{\mathrm{ex}}^{\prime \prime}$. However, after this delay the values of $h_{g}$ are remarkably independent of $\dot{q}_{e x}^{\prime \prime}$. This suggests that in modeling the burning of charring materials it may still be possible to decouple the pyrolysis analysis from the gas phase analysis.

\section{CONCLUSIONS}

A theoretical analysis for onedimensional pyrolysis of thermally thick materials shows that for charring materials the heat of gasification $h_{g}$ is no longer simply related to the heat of pyrolysis $h_{p}$.

Numerical solutions of the pyrolysis problem using material properties for cellulose were made for a steady incident heat flux. It is found that:

(i) The net flux into the solid decreases to 20 percent of its initial value because of surface heat loss. 
(ii) Pyrolysis occurs primarily in the temperature range $\mathrm{T}=600-750 \mathrm{~K}$; changes in reaction rate by a factor of 10 cause much smaller changes in $T_{w}, h_{p}$ and $h_{g}$.

(iii) Changes in the value assumed for the heat of reaction $h_{r, \infty}$ cause significant changes in $\mathrm{h}_{\mathrm{g}}$; additional research is needed in this area.

(iv) Although the rate of pyrolysis increases with increasing incident flux level $\dot{q}_{\mathrm{ex}}^{\prime \prime}$ ' the values of $\mathrm{h}_{\mathrm{g}}$ after an initial transient are nearly independent of $\mathrm{q}_{\mathrm{ex}}^{\prime \prime}$.

(v) After an initial transient, the value of $h_{g}$ for a semi-infinite slab remains nearly constant at about twice the value of $h_{p}$. Because the $1 \mathrm{~cm}$ half-thickness slab does not remain thermally thick, its value of $h_{g}$ decreases with time to values below $h_{p}$. (A similar decrease in $h_{g}$ occurs for vaporizing fuels which do not remain thermally thick.)

We believe these results show that the heat of gasification is a useful concept for modeling fires on charring fuels. However, unlike the heat of pyrolysis, its value cannot be determined from thermodynamic properties alone, but must either be calculated as in this paper or found from experiment. Further studies of both types are recommended.

\section{NOMENCLATURE}

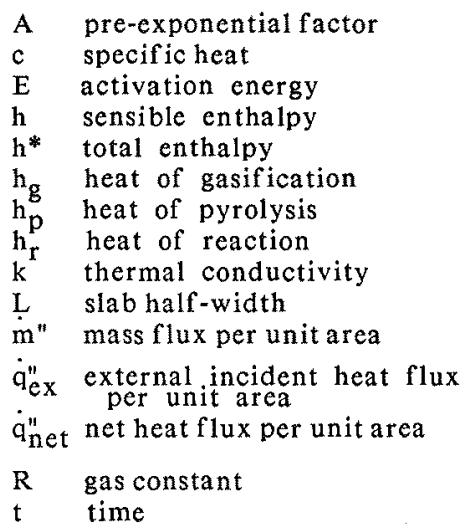

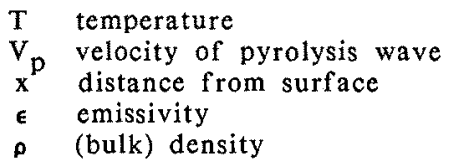

Subscripts

C final char value
$G$ gas (volatile products of pyrolysis)
$S$ initial solid value
$w$ at surface
$k$ char value during pyrolysis
$\sigma \quad$ active solid value during pyrolysis
$\infty \quad$ ambient

\section{ACKNOWLEDGEMENT}

It is a pleasure to acknowledge the assistance of Kenneth Siskind in carrying out the computations. This work was supported by the Center for Fire Research of the National Bureau of Standards under Grant NB83NADA4017.

\section{REFERENCES}

1. Delichatsios, M. A. and deRis, J. (1984). An analytical model for the pyrolysis of charring materials. Factory Mutual Research, Technical Report, J.I.OKOJ1.BU.

2. Kanury, A. M. and Blackshear, P. L., Jr. (1970). Some considerations pertaining to the problem of wood burning. Combustion Science and Technology $1,339$. 
3. Kung, H-C. (1972). A mathematical model of wood pyrolysis. Combustion and Flame $18,185$.

4. Kung, H-C. and Kalelkar, A. S. (1973), On the heat of reaction in wood pyrolysis. Combustion and Flame 20, 91.

5. Lewellen, P. C., Peters, W. A. and Howard, J. B. (1977). Cellulose pyrolysis kinetics and char formation mechanism. Sixteenth Symposium (International) on Combustion, The Combustion Institute, Pittsburgh, pp. 1471-1480.

6. Pagni, P. J. (1980). Diffusion Flame Analyses. Fire Safety Journal $\underline{3}, 273$.

7. Sibulkin, M., Kulkarni, A. K. and Annamalai, K. (1982). Burning on a vertical fuel surface with finite chemical reaction rate. Combustion and Flame 44, 187.

8. Sibulkin, M. and Tewari, S. S. (1985). Measurements of flaming combustion of pure and fire retarded cellulose. Combustion and Flame 59. 31.

9. Tamanini, F. (1976). A numerical model for one-dimensional heat conduction with pyrolysis in a slab of finite thickness. (in) Factory Mutual Research, Serial No. 21011.7, Appendix A.

Table 1. Base Values of Properties

$$
\begin{aligned}
\mathrm{c}_{\mathrm{S}} & =1500 \mathrm{~J} / \mathrm{kgK} \\
\mathrm{c}_{\mathrm{C}} & =1000 \mathrm{~J} / \mathrm{kgK} \\
\mathrm{c}_{\mathrm{p}, \mathrm{G}} & =1700 \mathrm{~J} / \mathrm{kgK} \\
\mathrm{k}_{\mathrm{S}} & =0.2 \mathrm{~W} / \mathrm{mK} \\
\mathrm{k}_{\mathrm{C}} & =0.08 \mathrm{~W} / \mathrm{mK} \\
\rho_{\mathrm{S}} & =500 \mathrm{~kg} / \mathrm{m}^{3} \\
{ }_{\mathrm{P}} & =150 \mathrm{~kg} / \mathrm{m}^{3} \\
\mathrm{E} & =1.5 \times 10^{8} \mathrm{~J} / \mathrm{kg} \text {-mole } \\
\mathrm{A} & =1 \times 10^{10} \mathrm{~s}^{-1} \\
\mathrm{~h}_{\mathrm{r}, \infty} & =-5 \times 10^{5} \mathrm{~J} / \mathrm{kg} \\
\epsilon & =1.0
\end{aligned}
$$

$\mathrm{L}=1 \mathrm{~cm}$ and $\dot{\mathrm{q}}_{\mathrm{ex}}^{\prime \prime}=50 \mathrm{~kW} / \mathrm{m}^{2}$ (except where noted).

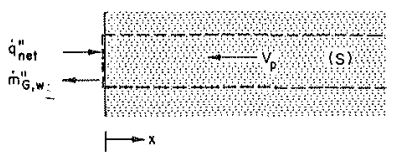

(a) Voporizing fue! (e.g.PMMA)

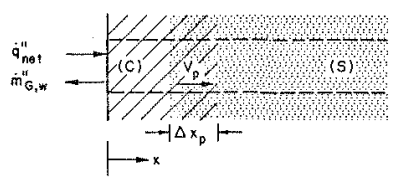

(b) Charring fuel (e.q, celitulase)

Fig. 1. Control volumes used in analysis of pyrolyzing fuels.

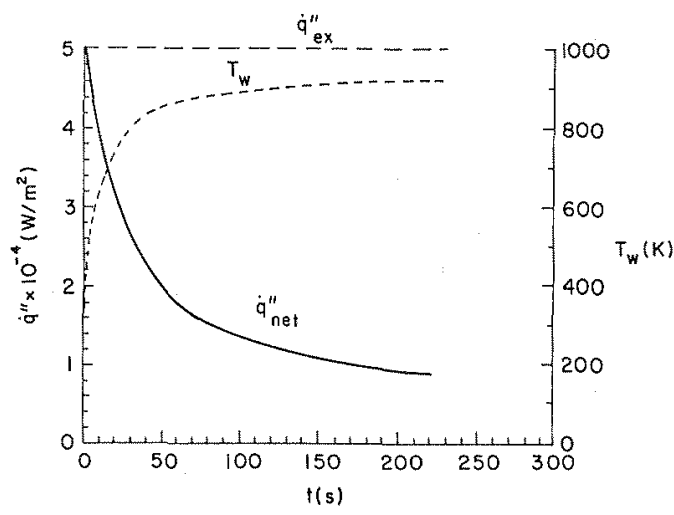

Fig. 2. Surface temperature response and net heat flux. $\mathrm{L}=1 \mathrm{~cm}$. 


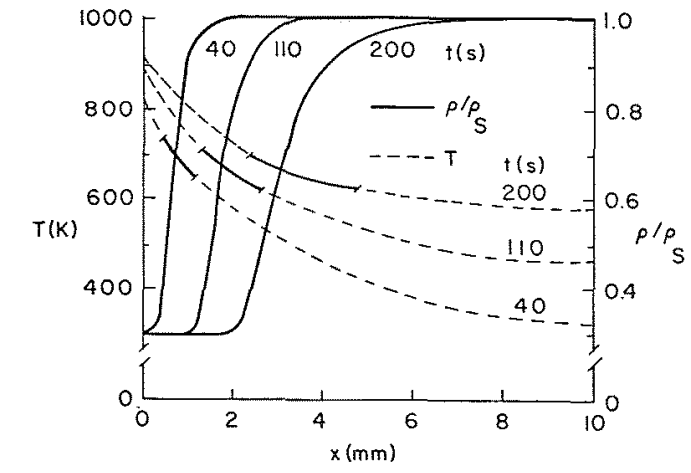

Fig. 3. Internal density and temperature $\stackrel{\omega}{\mathscr{\Xi}}$

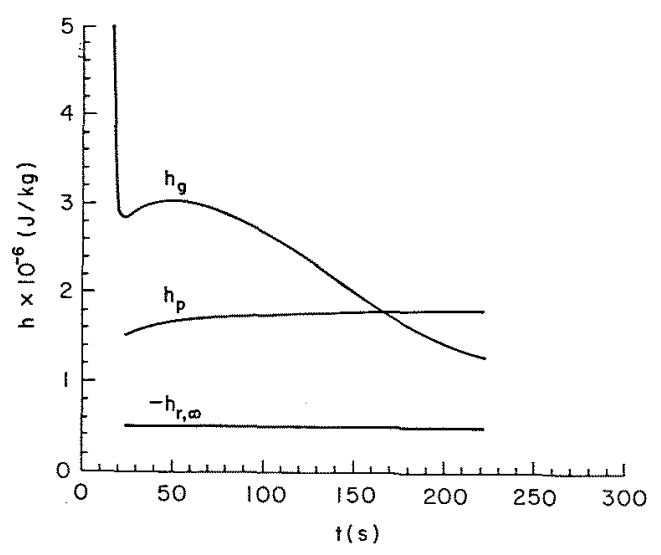

Fig. 5. Comparison of heat of reaction, pyrolysis and gasification.

$\mathrm{L}=1 \mathrm{~cm}$.

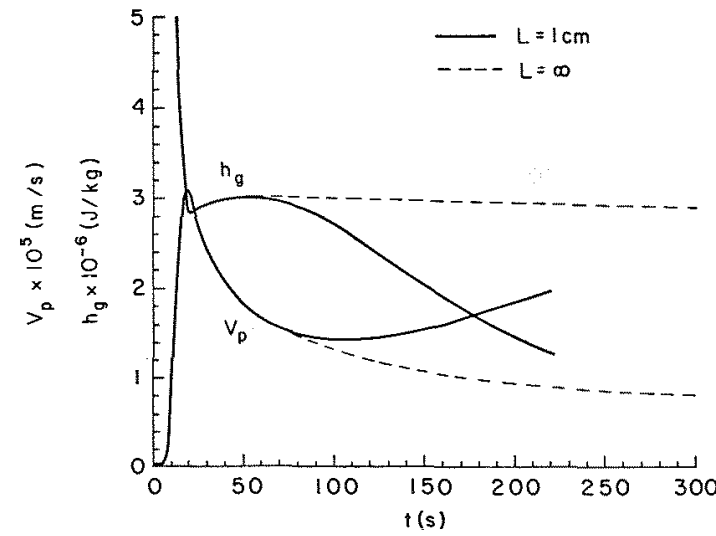

Fig. 4. Pyrolysis wave velocity and heat of gasification for base case ( $\mathrm{L}=1 \mathrm{~cm}$ ) and for semi-infinite slab $(\mathrm{L}=\infty)$.

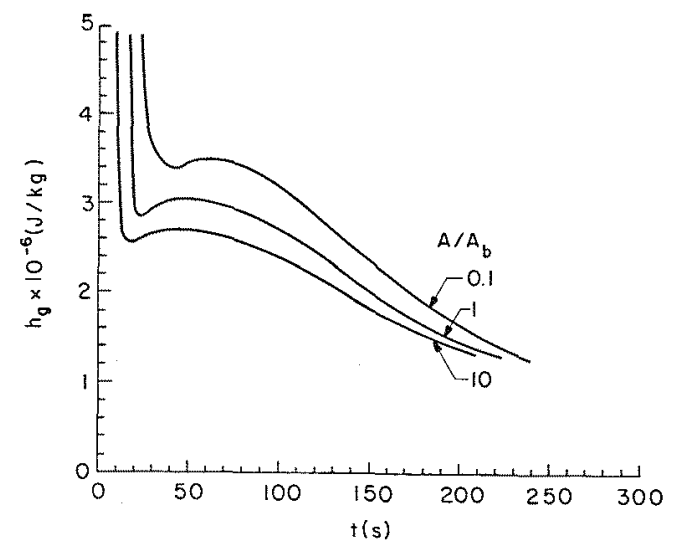

Fig. 6. Sensitivity of heat of gasification to rate of chemical reaction. $\mathrm{L}=1 \mathrm{~cm}$. 


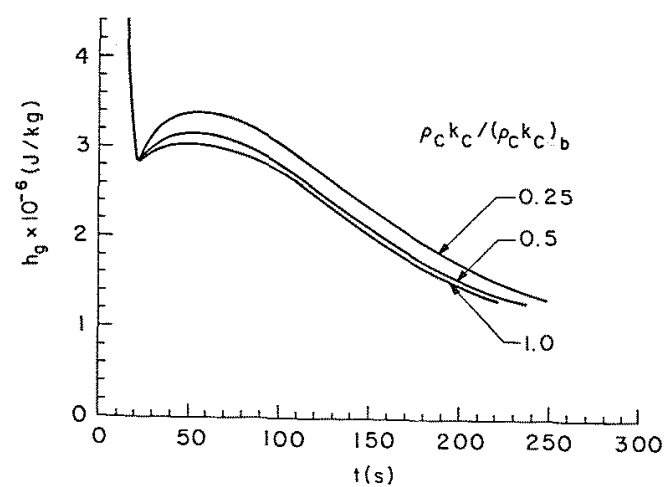

Fig. 7. Sensitivity of heat of gasification to properties of char. $L=1 \mathrm{~cm}$.

8

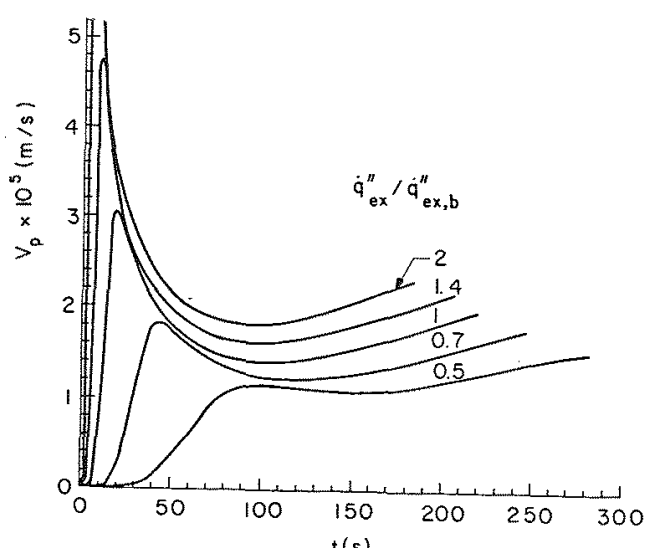

Fig. 9. Effect of external incident heat flux on pyrolysis wave velocity. $\mathrm{L}=1 \mathrm{~cm}$.

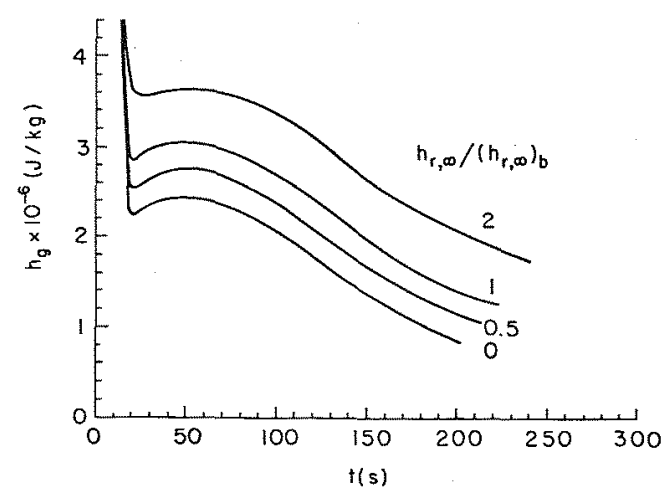

Fig. 8. Sensitivity of heat of gasification to value of heat of reaction. $\mathrm{L}=1 \mathrm{~cm}$.

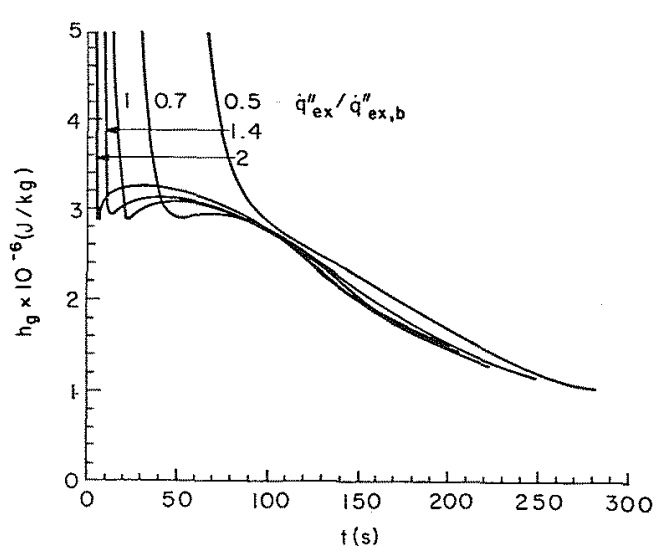

Fig. 10. Fffect of external incident heat flux on heat of gasification. $\mathrm{L}=1 \mathrm{~cm}$. 